\title{
Numerical calculation of the Hamada basis vectors for three-dimensional toroidal magnetic configurations
}

\author{
J. N. Talmadge and S. P. Gerhardt ${ }^{\text {a) }}$ \\ HSX Plasma Laboratory, University of Wisconsin-Madison, Madison, Wisconsin 53706
}

(Received 4 February 2005; accepted 2 May 2005; published online 1 July 2005)

\begin{abstract}
The moment equation approach to neoclassical transport is used to calculate neoclassical particle and heat fluxes, impurity transport, the ambipolar electric field, and momentum damping rates. These equations are often written in Hamada coordinates which makes it easier to obtain analytic solutions. However, previous simplifying assumptions used to evaluate the basis vectors analytically are often invalid for advanced stellarator configurations. In this paper, a numerical method is presented by which the Hamada basis set can be determined for an arbitrary three dimensional toroidal confinement device by integrating along a magnetic field line. The method is applied to the magnetic configuration in the Helically Symmetric Experiment [F. S. B. Anderson, A. F. Almagri, D. T. Anderson, P. G. Matthews, J. N. Talmadge, and J. L. Shohet, Fusion Technol. 27, 273 (1995)] and compared to the large-aspect-ratio tokamak approximation to the basis set. The results indicate that the numerical technique is a more accurate method to specify the basis vectors, especially in a device with negligible toroidal curvature. (C) 2005 American Institute of Physics.
\end{abstract}

[DOI: 10.1063/1.1938507]

\section{INTRODUCTION}

The moment equation approach to neoclassical theory is often used in toroidal systems to determine the neoclassical particle and heat fluxes, radial current, impurity transport as well as the time dependence and steady-state limit of the plasma rotation velocity, and ambipolar electric field. ${ }^{1-3}$ For plasmas in which the magnetic field is fully three dimensional, it is natural to write such equations using a flux coordinate system, such as Boozer ${ }^{4}$ or Hamada, ${ }^{5}$ in which the volume or toroidal flux acts as a radial coordinate and the other two coordinates are angles on the flux surface. Since it is often desired to compare the results of such a calculation with the results of an experiment, it has frequently been the case that some simplifying assumptions have been made regarding the nature of the basis vectors. In one paper, for example, the Hamada coordinates were approximated by cylindrical coordinates. ${ }^{6}$ Subsequently, Coronado and Galindo Trejo derived analytic expressions for the basis vectors that are appropriate for a large-aspect-ratio tokamak. ${ }^{7}$ These expressions were then used to calculate the rotation damping rates and radial conductivity for an arbitrary threedimensional toroidal geometry that includes the effects of both parallel viscosity and ion-neutral collisions. ${ }^{8}$

However, the validity of applying Hamada basis vectors derived for a tokamak directly to a three-dimensional stellarator needs to be investigated. Furthermore, knowledge of how the Hamada basis vectors relate to a laboratory coordinate system is necessary to understand the direction of plasma flow and momentum damping rates when subject to an external momentum source. It is one goal of a new generation of quasisymmetric stellarators with reduced parallel viscous damping to achieve improved confinement param-

\footnotetext{
${ }^{a)}$ Present address: Princeton Plasma Physics Laboratory, Princeton, New Jersey 08543.
}

eters. Such configurations may even have very little toroidal curvature such as a quasihelically symmetric configuration or a quasipoloidal device. This makes the suitability of the basis vectors derived for a tokamak even more problematic. The method outlined here has been used to demonstrate that parallel viscous damping in the Helically Symmetric Experiment $^{9}$ (HSX) is reduced compared to a configuration in which the quasisymmetry is intentionally broken. ${ }^{10}$

In this paper, we present a numerical technique for calculating the Hamada basis vectors for an arbitrary toroidal geometry. The technique employed is based, in part, on using the method of Nemov ${ }^{11}$ to calculate the gradient of a magnetic surface function in a complex magnetic geometry like a stellarator. This allows us the ability to integrate a set of differential equations along a magnetic field line using a realistic representation of the magnetic field components produced by a set of magnet coils as calculated from a BiotSavart code. We then apply this method to a calculation of the basis vectors for HSX. This is an interesting test of the tokamak approximation to the Hamada basis vectors because HSX is unique in being a toroidal stellarator with negligible toroidal curvature. ${ }^{12}$ Our paper is organized as follows: in Sec. II we present the general method for calculating numerically the Hamada basis vectors. Section III describes the method to obtain the initial conditions for the integration. Section IV summarizes the results of the analytic largeaspect-ratio tokamak approximation to the basis vectors, while in Sec. V we compare these results to the numerical calculation. Finally, in Sec. VI, we discuss the results and summarize the conclusions.

\section{EQUATIONS FOR THE HAMADA BASIS VECTORS}

The Hamada coordinates are given as $\{\psi, \alpha, \varsigma\}$ where $\psi$ is the toroidal flux through a magnetic surface, $\alpha$ is the Hamada poloidal angle, and $s$ is the Hamada toroidal angle. A 
vector field $\mathbf{A}$, where $\mathbf{A}$ can be the magnetic field $\mathbf{B}$ or the fluid velocity $\mathbf{U}$, for example, can be written in the Hamada coordinate system as

$$
\begin{aligned}
\mathbf{A} & =(\mathbf{A} \cdot \boldsymbol{\nabla} \psi) \mathbf{e}_{\psi}+(\mathbf{A} \cdot \boldsymbol{\nabla} \alpha) \mathbf{e}_{\alpha}+(\mathbf{A} \cdot \boldsymbol{\nabla} \varsigma) \mathbf{e}_{\varsigma} \\
& =A^{\psi} \mathbf{e}_{\psi}+A^{\alpha} \mathbf{e}_{\alpha}+A^{\varsigma} \mathbf{e}_{\boldsymbol{\varsigma}}
\end{aligned}
$$

or alternatively as

$$
\begin{aligned}
\mathbf{A} & =\left(\mathbf{A} \cdot e_{\psi}\right) \boldsymbol{\nabla} \psi+\left(\mathbf{A} \cdot \mathbf{e}_{\alpha}\right) \boldsymbol{\nabla} \alpha+\left(\mathbf{A} \cdot \mathbf{e}_{\mathrm{\varsigma}}\right) \boldsymbol{\nabla} \mathrm{s} \\
& =A_{\psi} \boldsymbol{\nabla} \psi+A_{\alpha} \boldsymbol{\nabla} \alpha+A_{\mathrm{\varsigma}} \boldsymbol{\nabla} \mathrm{s} .
\end{aligned}
$$

In the equations above $A^{\psi}=\mathbf{A} \cdot \boldsymbol{\nabla} \psi, A_{\psi}=\mathbf{A} \cdot \mathbf{e}_{\psi}$, and similar definitions exist for the other components of the vector. It is customary to call $\left\{\mathbf{e}_{\psi}, \mathbf{e}_{\alpha}, \mathbf{e}_{\varsigma}\right\}$ the covariant basis set and $\{\boldsymbol{\nabla} \psi, \boldsymbol{\nabla} \alpha, \boldsymbol{\nabla} \mathrm{s}\}$ the contravariant basis set. The two sets are reciprocal in that $\mathbf{e}_{\psi} \cdot \boldsymbol{\nabla} \psi=1, \mathbf{e}_{\psi} \cdot \boldsymbol{\nabla} \alpha=0, \mathbf{e}_{\psi} \cdot \boldsymbol{\nabla} \mathbf{s}=0$, etc., from which it follows that

$$
\mathbf{e}_{1}=\sqrt{g}\left(\boldsymbol{\nabla} u^{2} \times \boldsymbol{\nabla} u^{3}\right)
$$

and cyclic permutations of this relation exist to obtain the other covariant basis_vectors. In Hamada coordinates, the Jacobian, given by $\sqrt{g}$ in the expression above, is a fluxsurface constant and is given by

$$
\sqrt{g}=[\nabla \psi \cdot(\nabla \alpha \times \nabla \varsigma)]=\frac{1}{4 \pi^{2}} \frac{d V}{d \psi} .
$$

Here $V$ is the volume enclosed by the flux-surface and the angular coordinates go from 0 to $2 \pi$. In the Hamada coordinate system, both the magnetic field lines and the current density lines are straight. ${ }^{13}$

In this paper, we wish to derive a set of differential equations that can be used to solve for the components of the Hamada basis set. The contravariant basis set is given by the following three equations: ${ }^{13}$

$$
\begin{aligned}
& B^{\psi}=\mathbf{B} \cdot \nabla \psi=0, \\
& B^{\alpha}=\mathbf{B} \cdot \nabla \alpha=\frac{\boldsymbol{t}}{2 \pi \sqrt{g}}, \\
& B^{\varsigma}=\mathbf{B} \cdot \boldsymbol{\nabla} \zeta=\frac{1}{2 \pi \sqrt{g}},
\end{aligned}
$$

where $t$ is the rotational transform. The equations for the contravariant basis set are of the form

$$
B^{f}=\mathbf{B} \cdot \boldsymbol{\nabla} f=S_{f}(\psi),
$$

where $f=\{\psi, \alpha, \varsigma\}$ and $S_{f}=\{0, t / 2 \pi \sqrt{g}, 1 / 2 \pi \sqrt{g}\}$.

For a right-handed Hamada coordinate system, $\psi$ increases in the radial direction, $\alpha$ increases poloidally in the counterclockwise direction, and $s$ then increases clockwise in the toroidal direction looking down. We want to solve for the components of $\boldsymbol{\nabla} f$ in a cylindrical coordinate system that can be compared to measurements in a laboratory. The coordinates $\{R, Z, \phi\}$ describe such a right-handed system where $R$ and $Z$ are the usual cylindrical coordinates and the toroidal angle $\phi$ is in the same direction as the Hamada toroidal angle s. Following Nemov, and using similar notation, we define the components of $\nabla f$ as

$$
P_{f}=\frac{\partial f}{\partial R}, \quad G_{f}=\frac{\partial f}{\partial Z}, \quad Q_{f}=\frac{\partial f}{\partial \phi}
$$

so that Eq. (5) can be written as

$$
B_{R} P_{f}+B_{Z} G_{f}+\frac{B_{\phi}}{R} Q_{f}=S_{f}(\psi) .
$$

Taking the derivative of Eq. (7) with respect to $R, Z$, and $\phi$ we obtain the following magnetic differential equations for the components of $\nabla f$ :

$$
\begin{aligned}
& B_{R} \frac{\partial P_{f}}{\partial R}+B_{Z} \frac{\partial P_{f}}{\partial Z}+\frac{B_{\phi}}{R} \frac{\partial P_{f}}{\partial \phi} \\
& =\frac{\partial S_{f}}{\partial R}-\left[\frac{\partial B_{R}}{\partial R} P_{f}+\frac{\partial B_{Z}}{\partial R} G_{f}+\frac{\partial}{\partial R}\left(\frac{B_{\phi}}{R}\right) Q_{f}\right], \\
& B_{R} \frac{\partial G_{f}}{\partial R}+B_{Z} \frac{\partial G_{f}}{\partial Z}+\frac{B_{\phi}}{R} \frac{\partial G_{f}}{\partial \phi} \\
& =\frac{\partial S_{f}}{\partial Z}-\left[\frac{\partial B_{R}}{\partial Z} P_{f}+\frac{\partial B_{Z}}{\partial Z} G_{f}+\frac{\partial}{\partial Z}\left(\frac{B_{\phi}}{R}\right) Q_{f}\right], \\
& B_{R} \frac{\partial Q_{f}}{\partial R}+B_{Z} \frac{\partial Q_{f}}{\partial Z}+\frac{B_{\phi}}{R} \frac{\partial Q_{f}}{\partial \phi} \\
& =\frac{\partial S_{f}}{\partial \phi}-\left[\frac{\partial B_{R}}{\partial \phi} P_{f}+\frac{\partial B_{Z}}{\partial \phi} G_{f}+\frac{\partial}{\partial \phi}\left(\frac{B_{\phi}}{R}\right) Q_{f}\right],
\end{aligned}
$$

where we have used the following relationships:

$$
\frac{\partial Q_{f}}{\partial R}=\frac{\partial P_{f}}{\partial \phi}, \quad \frac{\partial G_{f}}{\partial R}=\frac{\partial P_{f}}{\partial Z}, \quad \frac{\partial G_{f}}{\partial \phi}=\frac{\partial Q_{f}}{\partial Z} .
$$

For each of the Hamada coordinates, $f=\{\psi, \alpha, \varsigma\}$, there are three equations that can be put in the form of Eqs. (8a)-(8c) for a total of nine equations that need to be solved.

Derivatives of the right-hand side of Eq. (7), $\partial S_{f} / \partial R$, $\partial S_{f} / \partial Z$, and $\partial S_{f} / \partial \phi$, can be obtained in the following manner, using $S_{\alpha}$ as an example:

$$
\frac{\partial S_{\alpha}}{\partial R}=\frac{1}{2 \pi \sqrt{g}}\left[\frac{d t}{d \psi}-\frac{t}{\sqrt{g}} \frac{d \sqrt{g}}{d \psi}\right] P_{\psi},
$$

where we have used the fact that the rotational transform and Jacobian are flux-surface functions and $P_{\psi}=\partial \psi / \partial R$. Similar equations can be derived for the partial derivatives $\partial S_{\alpha} / \partial \phi$, $\partial S_{\alpha} / \partial Z, \partial S_{\varsigma} / \partial R, \partial S_{\varsigma} / \partial Z$, and $\partial S_{\varsigma} / \partial \phi$. The derivatives of $S_{\psi}$ are all zero. The Jacobian, given in Eq. (3), is evaluated from the expression given by Solov'ev and Shafranov, ${ }^{14}$

$$
\frac{d V}{d \psi}=\lim _{N \rightarrow \infty} \frac{1}{N} \int \frac{d l}{B},
$$

where $N$ is the number of toroidal transits of a field line. Equations (8) and (11) can be cast in the form

$$
\text { B } \cdot \boldsymbol{\nabla} A=D,
$$

which can be solved from a set of three coupled ordinary differential equations ${ }^{11}$ 


$$
\begin{aligned}
& \frac{d R}{d \phi}=\frac{R B_{R}}{B_{\phi}}, \\
& \frac{d Z}{d \phi}=\frac{R B_{Z}}{B_{\phi}}, \\
& \frac{d A}{d \phi}=\frac{R}{B_{\phi}} D .
\end{aligned}
$$

The first two expressions above are just the equations for a magnetic field line. Thus using the derivatives of the transform and the Jacobian with respect to flux, the nine equations corresponding to Eq. (10) (three of which are identically zero), and the set of nine differential equations of Eq. (8) recast in the form of Eqs. (13), the entire Hamada basis set can be computed. What remains is to obtain a set of initial conditions for the basis vectors.

\section{INITIAL CONDITIONS}

Each of the components of the contravariant angle coordinates must be periodic on a flux surface. For magnetic differential equations of the form given in Eq. (12), B $\boldsymbol{\nabla} A$ $=D$, it was shown by Newcomb ${ }^{15}$ that for $A$ to be single valued, on every closed field line on a rational surface the condition $\oint(d l / B) D=0$ must be satisfied. Because of the lack of symmetry in a stellarator, this condition may not be satisfied. However, for the purpose of this calculation, which is to help make comparisons between experimental results and a theoretical model, we are interested in flux surfaces where the rotational transform is irrational. Therefore, a condition necessary for solvability will be applied, $\langle\mathbf{B} \cdot \boldsymbol{\nabla} A\rangle=\langle D\rangle=0$, where the brackets indicate an average over the flux surface. This condition has been used previously to ensure that the potential derived from the Pfirsch-Schlüter current is a single-valued function of position. ${ }^{16,17}$ In this paper we will use this same condition to force the contravariant components to be single valued after first obtaining a set of constraints on the initial conditions and then a relation between the basis vectors and the Pfirsch-Schlüter current.

There are nine initial conditions that have to be satisfied to obtain the basis vectors: $P_{\psi}=\partial \psi / \partial R, G_{\psi}=\partial \psi / \partial Z, Q_{\psi}$ $=\partial \psi / \partial \phi, P_{\alpha}=\partial \alpha / \partial R, G_{\alpha}=\partial \alpha / \partial Z, Q_{\alpha}=\partial \alpha / \partial \phi, P_{\varsigma}=\partial \varsigma / \partial R$, $G_{\varsigma}=\partial \varsigma / \partial Z, Q_{\varsigma}=\partial \varsigma / \partial \phi$. A simplifying set of initial conditions can be specified if we take advantage of the two locations in each field period where the magnetic surfaces are symmetric about the $R$ axis. At these locations, along $Z=0$, the radial component of the magnetic field $B_{R}=0$ and the normal to the surface points in the $\hat{R}$ direction. Thus we can calculate numerically the derivative of the toroidal flux with respect to $R$, $P_{\psi}$, and set $Q_{\psi}=G_{\psi}=0$. Again, invoking the symmetry along the line $Z=0$, we can set the initial values of $\alpha$ and $s$ to be constant on the axis so that $P_{\alpha}=P_{\mathrm{s}}=0$. All together, we have the first five initial conditions:

$$
P_{\psi}=\frac{\partial \psi}{\partial R}, \quad Q_{\psi}=G_{\psi}=P_{\alpha}=P_{\varsigma}=0 .
$$

Three additional initial conditions come from the constraints on $\mathbf{B} \cdot \boldsymbol{\nabla} \alpha, \mathbf{B} \cdot \boldsymbol{\nabla}$ s, and the Jacobian $\sqrt{g}$ from Eqs. (3), (4b), and (4c). Making use of Eq. (14) and the fact that $B_{R}$ $=0$ along $Z=0$ at the symmetry plane then

$$
\begin{aligned}
& B_{Z} G_{\alpha}+B_{\phi} \frac{Q_{\alpha}}{R}=\frac{2 \pi t}{\frac{d V}{d \psi},} \\
& B_{Z} G_{\varsigma}+B_{\phi} \frac{Q_{\varsigma}}{R}=\frac{2 \pi}{\frac{d V}{d \psi}}, \\
& G_{\alpha} Q_{\varsigma}-Q_{\alpha} G_{\varsigma}=\frac{R \frac{d V}{d \psi}}{4 \pi P_{\psi}} .
\end{aligned}
$$

One more initial condition is needed; this condition ensures that the Hamada angle basis vectors are periodic. It follows from a correspondence between one of the basis vectors and the Pfirsch-Schlüter current and a numerical calculation of this current as given by Nemov. ${ }^{17}$ The PfirschSchlüter current can be written as

$$
\mathbf{J}_{\|}=h \mathbf{B} \frac{d p}{d \psi},
$$

where $h$ is obtained by solving the magnetic differential equation

$$
\mathbf{B} \cdot \boldsymbol{\nabla} h=\frac{2}{B^{3}}(\mathbf{B} \times \nabla \psi) \cdot \boldsymbol{\nabla} B .
$$

The initial condition $h_{0}$ for $h$ is obtained by requiring that the potential defined by $-\nabla \Phi=\mathbf{J}_{\|} / \sigma_{\|}$be single valued. This is equivalent to having

$$
\langle\mathbf{B} \cdot \boldsymbol{\nabla} \Phi\rangle=-\frac{d p}{d \psi} \frac{1}{\sigma_{\|}}\left\langle\left(h_{0}+h\right) B^{2}\right\rangle=0,
$$

where the brackets indicate an average over the flux surface. If we write $\Phi=\Phi_{1}+h_{0} \Phi_{2}$, then the single-valued nature of the potential is forced when the initial condition satisfies $h_{0}$ $=-\Phi_{1} / \Phi_{2}$, where $\Phi_{1}$ and $\Phi_{2}$ are calculated from

$$
\begin{aligned}
& \text { B } \cdot \nabla \Phi_{1}=-h B^{2}, \\
& \text { B } \cdot \nabla \Phi_{2}=-B^{2} .
\end{aligned}
$$

The initial condition for $h$ then allows for the final initial condition for the basis vectors when we use the expression obtained by Coronado and Wobig ${ }^{18}$ for the parallel current

$$
\mathbf{J}_{\|}=\frac{B_{\alpha} \mathbf{B}}{B^{\varsigma} B^{2} \sqrt{g}} \frac{d p}{d \psi}+\Lambda(\psi) \mathbf{B} .
$$

In the equation above, $\Lambda(\psi)$ is a constant that represents the plasma currents whose flux-surface average is not equal to zero, such as the bootstrap or ohmic current, and the first term represents the Pfirsch-Schlüter current.

If we equate Eq. (16) to the first term in Eq. (20) then we can obtain the following equation: 


$$
B_{\alpha}=\mathbf{B} \cdot \mathbf{e}_{\alpha}=h B^{\varsigma} B^{2} \sqrt{g}=2 \pi h B^{2},
$$

where we have made use of Eq. (4c). Using $\mathbf{e}_{\alpha}=\sqrt{g}(\boldsymbol{\nabla}$ s $\times \nabla \psi$ ), inserting Eq. (14) into Eq. (21) and noting again that $B_{R}=0$ at the symmetry plane, we can obtain the final initial condition for the basis vectors

$$
B_{Z} \frac{Q_{\varsigma}}{R}-B_{\phi} G_{\varsigma}=\frac{h_{0} B^{2}}{P_{\psi}} \frac{2 \pi}{\frac{d V}{d \psi}} .
$$

Here $h_{0}$ is the initial condition on $h$, as defined by Eqs. (19a) and (19b). Using Eq. (15b) along with Eq. (22), we have two simultaneous equations for $Q_{\varsigma}$ and $G_{\varsigma}$. With those results, we can then solve for $Q_{\alpha}$ and $G_{\alpha}$ using Eqs. (15a) and (15c) to finally derive

$$
\begin{aligned}
& Q_{\varsigma}=\left[\frac{h_{0} R B_{Z}}{P_{\psi}}+\frac{R B_{\phi}}{B^{2}}\right] \frac{2 \pi}{\frac{d V}{d \psi}}, \\
& G_{\varsigma}=\left[\frac{B_{Z}}{B^{2}}-\frac{B_{\phi} h_{0}}{P_{\psi}}\right] \frac{2 \pi}{\frac{d V}{d \psi}}, \\
& Q_{\alpha}=\left[\frac{Q_{\varsigma} t-2 \pi B_{Z} / P_{\psi}}{B_{Z} G_{\varsigma}+B_{\phi} Q_{\varsigma} / R}\right] \frac{2 \pi}{\frac{d V}{d \psi}}, \\
& G_{\alpha}=\left[\frac{G_{\varsigma} t+2 \pi B_{\phi} / P_{\psi}}{B_{Z} G_{\varsigma}+B_{\phi} Q_{\varsigma} / R}\right] \frac{2 \pi}{\frac{d V}{d \psi}} .
\end{aligned}
$$

To summarize, the procedure that we use to obtain the contravariant basis vectors is first to numerically calculate, using a Biot-Savart code, the toroidal flux $\psi$, the rotational transform $t$, and the specific flux volume $d V / d \psi$ using Eq. (11). This is done for a set of neighboring flux surfaces in order to compute $P_{\psi}=d \psi / d R$ for Eq. (14) as well as $d t / d \psi$ and $d / d \psi(d V / d \psi)$ for the right-hand side of Eq. (8) as in the example of Eq. (10). The set of equations given by Eqs. (19a) and (19b) are then solved to obtain the initial condition $h_{0}$. With $h_{0}, P_{\psi}$, and $d V / d \psi$ and the components of the magnetic field on a flux surface at the symmetry plane, we can then compute the initial conditions given by Eqs. (23a)-(23d). It is then a matter of integrating the nine equations represented by Eqs. (8) for $f=\{\psi, \alpha, \varsigma\}$. Furthermore, we can compute the covariant basis set from Eq. (2). One last quantity that is very useful is the flux-surface average of the dot product of two basis vectors, where the flux-surface average of a function $A$ is defined by

$$
\langle A\rangle=\frac{\int \frac{d l}{B} A}{\int \frac{d l}{B}} .
$$

These surface averages are obtained by solving for $Y_{1} / Y_{2}$, where $\mathbf{B} \cdot \boldsymbol{\nabla} Y_{1}=A$ and $\mathbf{B} \cdot \boldsymbol{\nabla} Y_{2}=1$. In the following section we summarize the results of the large-aspect-ratio tokamak approximation to the Hamada basis set to which we want to compare the numerical calculation.

\section{LARGE-ASPECT-RATIO TOKAMAK APPROXIMATION}

The numerically calculated basis vectors for HSX will be compared to analytic expressions obtained by Coronado and Galindo Trejo ${ }^{7}$ using a large-aspect-ratio tokamak approximation. They assume a magnetic field in the form

$$
\mathbf{B}=\frac{B_{0}}{1+\varepsilon \cos \theta}[\Theta(r) \hat{\theta}+\hat{\phi}],
$$

where $r$ is the minor radius, $\theta$ is the poloidal angle, and $\phi$ is the toroidal angle. The function $\Theta=\varepsilon / q(r)$, where $\varepsilon=r / R_{0}$ is the inverse aspect ratio and $q(r) \equiv 1 / t$ is the safety factor. In this paper, we define the poloidal and toroidal Hamada coordinates $\alpha$ and s, to vary between 0 and $2 \pi$, not between 0 and 1 as in Ref. 7. Also, we use the flux function $\psi$ as the Hamada radial coordinate rather than the volume. This sets the Jacobian on each flux surface to be the same as in Eq. (3). Finally, we convert the results in Ref. 7 to the same $R, Z$, $\phi$ coordinate system in which the basis vectors for HSX are calculated, rather than the original $r, \theta, \phi$. The toroidal angle $\phi$ in both coordinate systems increases in the clockwise direction, looking down. Using this convention, the rotational transform in HSX is negative and ranges from -1.05 on the plasma axis to -1.12 at the edge.

If we keep only terms up to order $\varepsilon$, then the Jacobian for the magnetic field given by Eq. (25) is $R_{0} / 2 \pi B_{0}$ and the basis vectors from Ref. 7 can be rewritten in the following form:

$\boldsymbol{\nabla} \psi=2 B_{0} \pi r[\cos \theta \hat{R}+\sin \theta \hat{Z}]$,

$\boldsymbol{\nabla} \alpha=\frac{1}{r}[-\sin \theta \hat{R}+(\cos \theta+\varepsilon) \hat{Z}]$,

$\boldsymbol{\nabla} \boldsymbol{\varsigma}=\frac{1}{R_{0}}\left\{\left[2 \frac{r}{q} \frac{d q}{d r} \cos \theta-\frac{\varepsilon}{2}\left(1+\frac{r}{q} \frac{d q}{d r} \cos ^{2} \theta\right)\right] q \sin \theta \hat{R}\right.$ $+\left[2\left(1+\frac{r}{q} \frac{d q}{d r} \sin ^{2} \theta\right)-\frac{\varepsilon}{2} \cos \theta\left(1+\frac{r}{q} \frac{d q}{d r} \sin ^{2} \theta\right)\right] q \hat{Z}$ $+[1-\varepsilon \cos \theta] \hat{\phi}\}$,

$\mathbf{e}_{\psi}=\frac{1}{2 \pi B_{0} r}\left\{\left[\cos \theta+\varepsilon \sin ^{2} \theta\right] \hat{R}+[\sin \theta-\varepsilon \sin \theta \cos \theta] \hat{Z}\right.$ $\left.-\left[2\left(1+\frac{r}{q} \frac{d q}{d r}\right)-\left(1-\frac{3}{2} \frac{r}{q} \frac{d q}{d r}\right) \varepsilon \cos \theta\right] q \sin \theta \hat{\phi}\right\}$,

$\mathbf{e}_{\alpha}=r\{[-(1-\varepsilon \cos \theta) \sin \theta] \hat{R}+[(1-\varepsilon \cos \theta) \cos \theta] \hat{Z}$

$$
\left.-\left[2 \cos \theta+\varepsilon / 2\left(1-2 \cos ^{2} \theta\right)\right] q \hat{\phi}\right\},
$$




$$
\mathbf{e}_{\mathrm{s}}=R_{0}[1+\varepsilon \cos \theta] \hat{\phi} \text {. }
$$

In the moment approach, the first-order momentum balance equation is simplified by averaging over a flux surface the dot product of the equation with the magnetic field. A number of terms then drop out since for any scalar $s$, the quantity $\langle\mathbf{B} \cdot \nabla s\rangle=0$. However, this also results in terms such as $\left\langle\mathbf{e}_{\alpha} \cdot \mathbf{e}_{\alpha}\right\rangle,\left\langle\mathbf{e}_{\alpha} \cdot \mathbf{e}_{\varsigma}\right\rangle$, etc. ${ }^{8}$ In the large-aspect-ratio tokamak approximation, where the flux surfaces are defined by $r$ $=$ const, the surface average of a function $F(\theta, \phi)$ is given by

$$
\langle F\rangle=\frac{1}{4 \pi^{2}} \int_{0}^{2 \pi} d \theta \int_{0}^{2 \pi} d \phi F(\theta, \phi)\left(1+\frac{r}{R_{0}} \cos \theta\right) .
$$

To order $\varepsilon$, the flux-surface averages of the dot products of the covariant and contravariant basis vectors are

$$
\begin{aligned}
& \langle\nabla \psi \cdot \nabla \psi\rangle=\left(2 B_{0} \pi r\right)^{2}, \\
& \langle\nabla \alpha \cdot \nabla \alpha\rangle=\frac{1}{r^{2}}, \\
& \langle\nabla \boldsymbol{\nabla} \cdot \boldsymbol{\nabla} \varsigma\rangle=\frac{1}{R_{0}^{2}}\left[1+4 q^{2}\left(1+\frac{r}{q} \frac{d q}{d r}+\frac{1}{2}\left\{\frac{r}{q} \frac{d q}{d r}\right\}^{2}\right)\right], \\
& \left\langle\mathbf{e}_{\psi} \cdot \mathbf{e}_{\psi}\right\rangle=\frac{1}{4 \pi^{2} B_{0} r^{2}}\left(1+2 q^{2}\left\{1+\frac{r}{q} \frac{d q}{d r}\right\}\right), \\
& \left\langle\mathbf{e}_{\alpha} \cdot \mathbf{e}_{\alpha}\right\rangle=r^{2}\left(1+2 q^{2}\right), \\
& \left\langle\mathbf{e}_{\varsigma} \cdot \mathbf{e}_{\varsigma}\right\rangle=R_{0}^{2}, \\
& \left\langle\mathbf{e}_{\alpha} \cdot \mathbf{e}_{\varsigma}\right\rangle=-2 q r^{2} .
\end{aligned}
$$

Also of interest when calculating plasma flow damping, for example, are the contravariant components of the magnetic field, $B^{\alpha}=\mathbf{B} \cdot \nabla \alpha$ and $B^{\varsigma}=\mathbf{B} \cdot \nabla \boldsymbol{s}$ which are constant on a magnetic surface, and the flux-surface average of the covariant components $\left\langle B_{\alpha}\right\rangle=\left\langle\mathbf{B} \cdot \mathbf{e}_{\alpha}\right\rangle$ and $\left\langle B_{\mathrm{\varsigma}}\right\rangle=\left\langle\mathbf{B} \cdot \mathbf{e}_{\varsigma}\right\rangle$,

$$
\begin{aligned}
& B^{\alpha}=\frac{B_{0}}{q R_{0}}, \\
& B^{\varsigma}=\frac{B_{0}}{R_{0}}, \\
& \left\langle B_{\alpha}\right\rangle=\frac{r^{2} B_{0}}{R_{0} q}, \\
& \left\langle B_{\varsigma}\right\rangle=R_{0} B_{0} .
\end{aligned}
$$

In the following section we will compare the numerical calculation of the Hamada basis vectors with the largeaspect-ratio tokamak approximation.

\section{CALCULATION OF THE HAMADA BASIS VECTORS FOR HSX}

HSX is a quasihelically symmetric stellarator, which to a good approximation means that the magnetic field is dominated by a single helical component $[n, m]=[4,1]$ in the magnetic field spectrum in addition to the $[0,0]$ term

$$
B=B_{0}\left[1-\delta_{4,1} \cos (4 \phi-1 \theta)\right] .
$$

There are also smaller amplitude symmetry-breaking terms in the spectrum, not shown in Eq. (30), that are less than $1 \%$ of the main field at the plasma edge, but included in the numerical calculation of the basis vectors nevertheless. Note that at $\phi=0^{\circ}$, the magnitude of $B$ varies as in a tokamak, with the high field on the inboard side and the low field on the outboard side. However, at the half-field period location, $\phi=45^{\circ}$, the high field side is on the outboard side of the torus. To compare the basis vectors in HSX to the equivalent tokamak, we assume a rotational transform profile for the tokamak that is identical to that in HSX. Also, we define the equivalent flux surface in the tokamak as $r=\sqrt{\psi / \pi B_{0}}$, where $\psi$ is the toroidal flux through a magnetic surface in HSX. Figure 1 shows a comparison between the components of the contravariant Hamada basis set, $\nabla \psi, \nabla \alpha, \nabla \mathrm{s}$ in HSX at the two toroidal locations $\phi=0^{\circ}$ and $\phi=45^{\circ}$, with the values in an axisymmetric tokamak. This is for a flux surface at $r / a$ $\sim 0.5$ and the components are plotted as a function of the Hamada poloidal angle $\alpha$.

Each of the components is single valued, validating the method for finding the initial conditions based on the Pfirsch-Schlüter current. Note that there is a significant difference in the components of the basis vectors for the two locations in HSX and that these components are also different from the large-aspect-ratio approximation. One readily apparent difference is that the components of the basis vectors in the toroidal direction, $Q_{\psi} / R$ and $Q_{\alpha} / R$, are identically zero for the tokamak approximation, but nonzero for the HSX numerical calculation. Furthermore, $Q_{\alpha} / R$ changes sign over one-half field period in HSX. Some terms, such as $P_{\mathrm{s}}$ and $Q_{\varsigma} / R$ agree reasonably well with the tokamak approximation, while other terms such as $P_{\alpha}, G_{\alpha}$, and $G_{\mathrm{s}}$ show a substantial variation as a function of toroidal angle.

Figure 2 shows the components of the covariant basis set, $\mathbf{e}_{\psi}, \mathbf{e}_{\alpha}, \mathbf{e}_{\mathrm{s}}$ in HSX compared to the analytic tokamak calculation. From the figure it can be seen that for the tokamak approximation, the components of $\mathbf{e}_{\mathrm{s}}$ in the $\hat{R}$ and $\hat{Z}$ directions are zero, whereas for HSX, these components are nonzero and change sign over one-half field period. Similarly for the $\mathbf{e}_{\alpha}$ vector, the component in the $\hat{\phi}$ direction also reverses in HSX at the two toroidal locations. Figure 3 shows more clearly how the numerical calculation of the basis vectors differs in magnitude and direction from each other at the two toroidal locations as well as from the analytic tokamak approximation. These plots are in the $Z-\phi$ plane at the outboard location on the midplane. Often the basis vectors do not even point in the same $Z$ or $\phi$ direction as the tokamak approximation. It is interesting to observe that at the plane in HSX for which the high magnetic field is on the outboard side of the device, the covariant basis vector $\mathbf{e}_{\alpha}$ is actually 

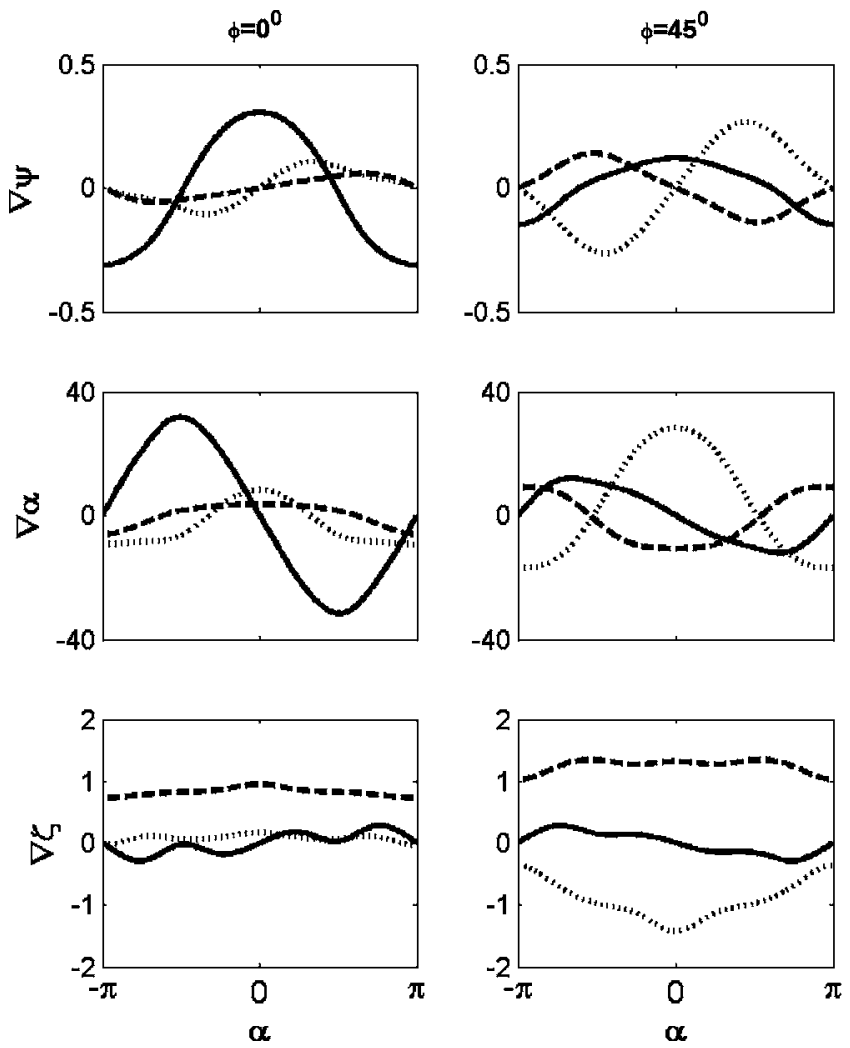

closer in magnitude and direction to the tokamak approximation than for the toroidal location in $\operatorname{HSX}$ where $|B|$ is more tokamak-like.

Figure 4 shows the radial profile of the surface average dot product of the basis vectors in HSX compared to the tokamak approximation from Eqs. (28a)-(28g). While the numerically calculated local basis vector may be pointing in
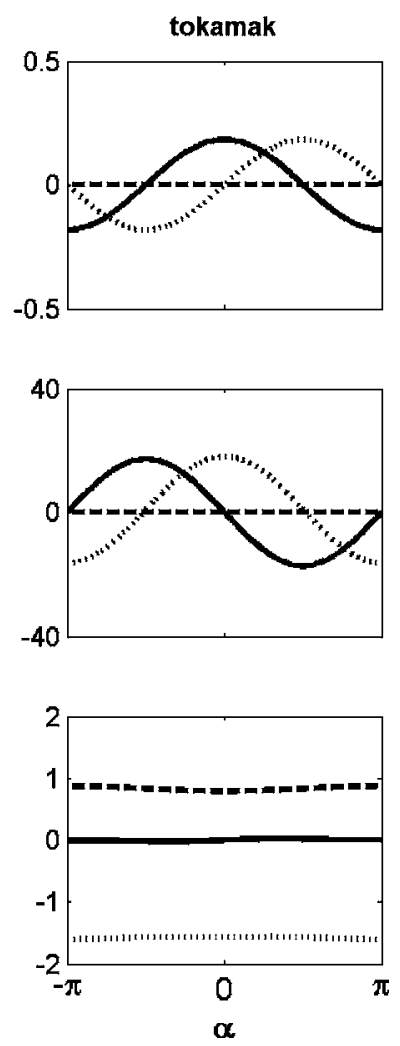

FIG. 1. The components of the contravariant basis set in HSX for a flux surface with $r / a \sim 0.5$ at toroidal locations $\phi=0^{\circ}$ and $\phi=45^{\circ}$, as well as for the equivalent tokamak based on the large-aspect-ratio approximation given in Ref. 7. The curves correspond to the component of the vector in the $\hat{R}$ ( $P$, solid line), $\hat{Z}$ ( $G$, dotted line), and $\hat{\phi}(Q / R$, dashed line) directions, and are plotted as a function of the Hamada poloidal angle $\alpha$.
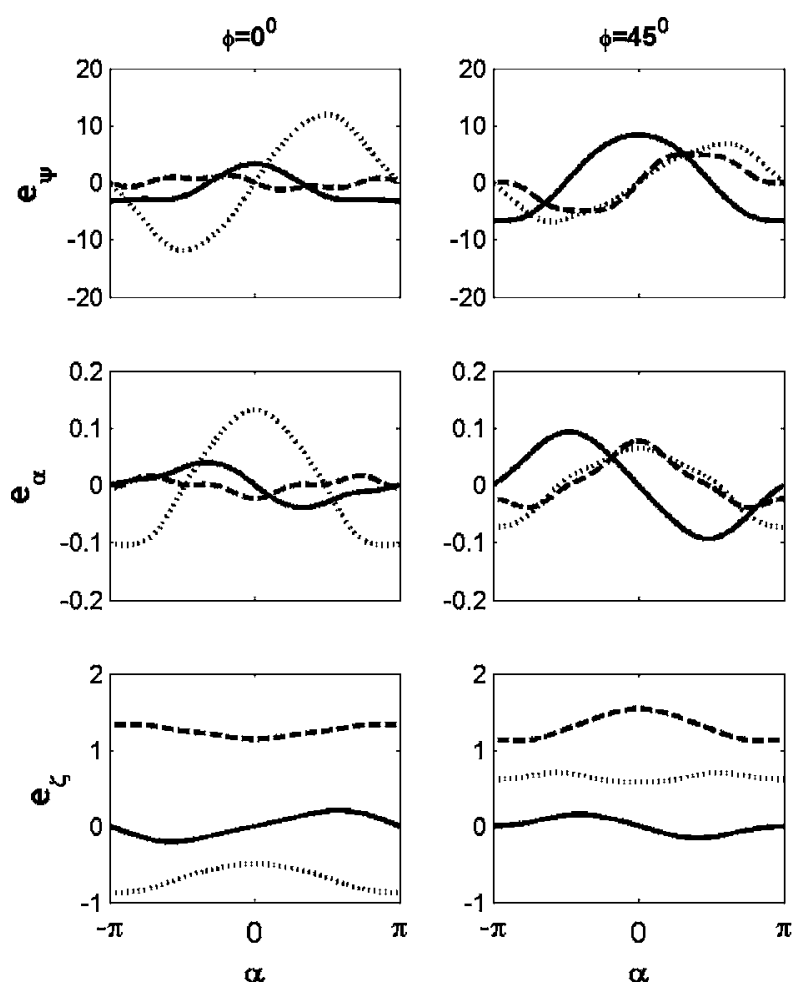
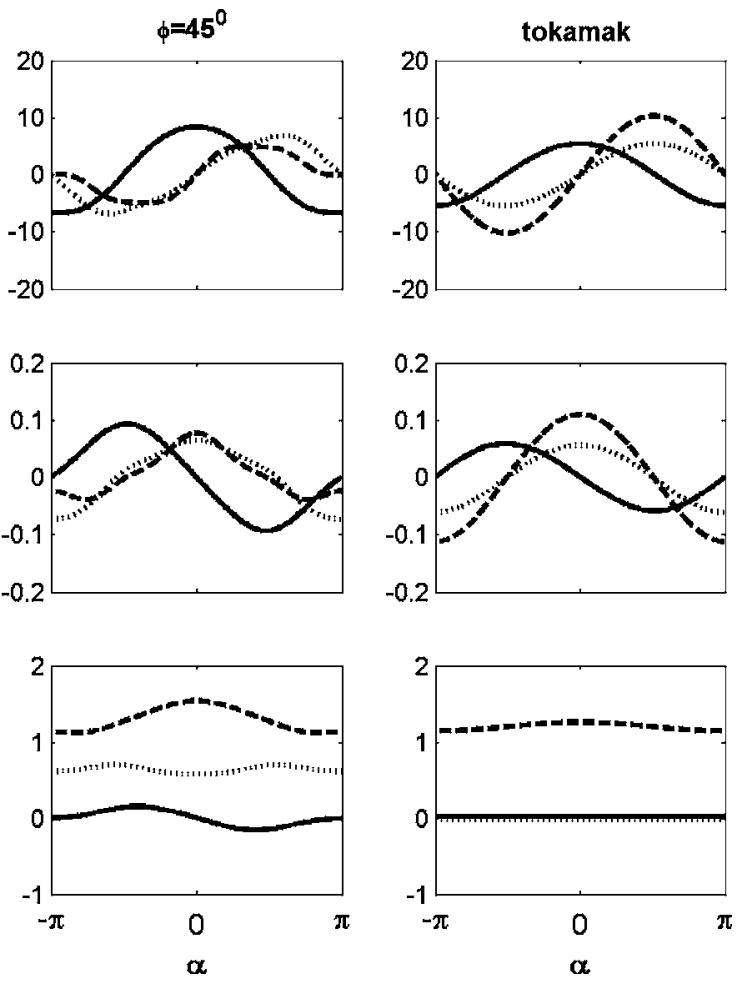

a different direction from the analytic result, the surface average of the basis vectors are similar in profile and differ at most by about $50 \%$. The analytic quantity $\langle\nabla \mathrm{s} \cdot \nabla \mathrm{s}\rangle$ decreases with radius while the numerical calculation shows the opposite trend. The surface average $\left\langle\mathbf{e}_{\alpha} \cdot \mathbf{e}_{\mathrm{s}}\right\rangle$ is negative for both the numerical and analytic calculations. Numerically 

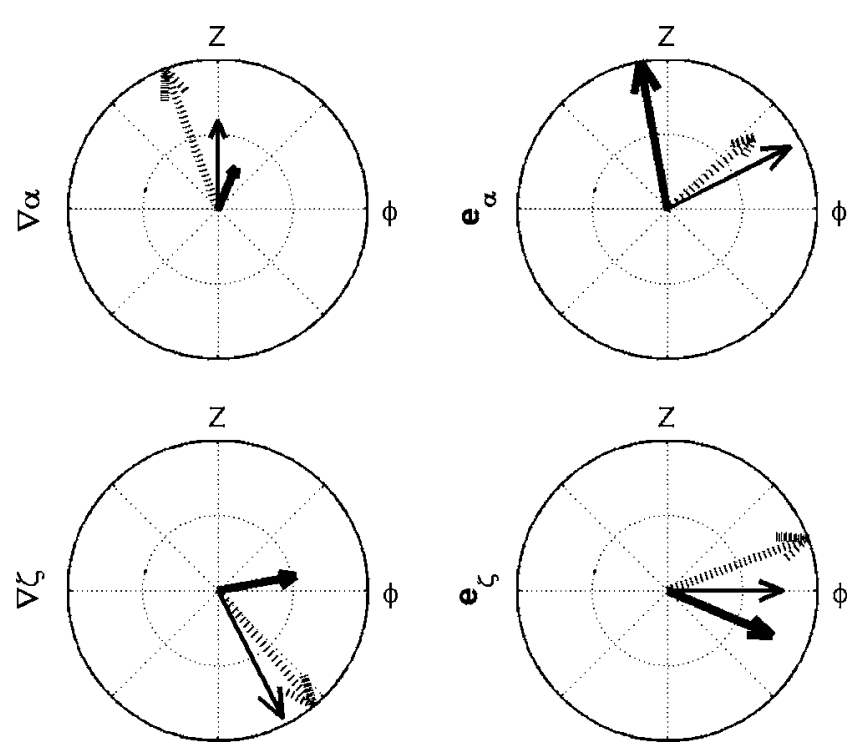

FIG. 3. Comparison in the $Z-\phi$ plane of the numerical calculation of the basis vectors at the outboard midplane at $\phi=0^{\circ}$ (thick solid line) and $\phi$ $=45^{\circ}$ (thick dotted line) with the analytic tokamak approximation (thin solid line).

calculated terms such as $\langle\nabla \alpha \cdot \nabla \alpha\rangle$ and $\left\langle\mathbf{e}_{\psi} \cdot \mathbf{e}_{\psi}\right\rangle$ show the $1 / r^{2}$ dependence of the analytic calculation. Finally Fig. 5 shows the radial profile of the contravariant components $B^{\alpha}$ and $B^{\varsigma}$ as well as the surface average values $\left\langle B_{\alpha}\right\rangle$ and $\left\langle B_{\varsigma}\right\rangle$. Note that there are substantial differences between the numerical and the analytic calculations. Another striking dissimilarity is that $\left\langle B_{\alpha}\right\rangle$ is identically equal to zero in the numerical calculation, but finite and increasing with radius in the analytic approximation. We will discuss why this is so, as well as some other interesting features of the calculations in the following section.

\section{DISCUSSION AND CONCLUSIONS}

It can be seen from Eq. (21) that the basis vector $\mathbf{e}_{\alpha}$ is related to $B_{\alpha}=\mathbf{B} \cdot \mathbf{e}_{\alpha}$ and the Pfirsch-Schlüter current. Also, from Fig. 3 it can be seen that the large-aspect-ratio tokamak approximation to this basis vector fits the magnitude and direction of the numerically calculated value at the half-field period better than at $\phi=0^{\circ}$. In this section, we will discuss these results further and make some summarizing conclusions.

In Fig. 6, $B_{\alpha}$ as well as the Pfirsch-Schlüter current [normalized to the pressure gradient, $\left.J_{\|} / d p / d \psi=\left(2 \pi B_{\alpha} / B\right)\right]$ are plotted as a function of the Hamada poloidal angle $\alpha$. Both $B_{\alpha}$ and $J_{\|}$reverse sign going from $\phi=0^{\circ}$ to $\phi=45^{\circ}$, whereas the tokamak result is independent of toroidal angle. This reflects the dominance of the $[4,1]$ component in the magnetic field spectrum for HSX, compared to $[0,1]$ term for the tokamak. In HSX, the dipole Pfirsch-Schlüter current rotates as a function of toroidal angle.

It should be noted that when we make comparisons between HSX and a tokamak, we are not comparing devices with the same amplitude of the dominant magnetic field component (besides the $[0,0]$ term). We have defined the equivalent flux surface in a tokamak to have a minor radius $r=\sqrt{\psi / \pi B_{0}}$, where $\psi$ is the toroidal flux through a surface in HSX. For the surface at $r / a \sim 0.5, \psi=0.00534 \mathrm{~Wb}$, corresponding to an effective radius of $0.0583 \mathrm{~m}$. With a major radius of $1.2 \mathrm{~m}$, this corresponds to a toroidal curvature com-
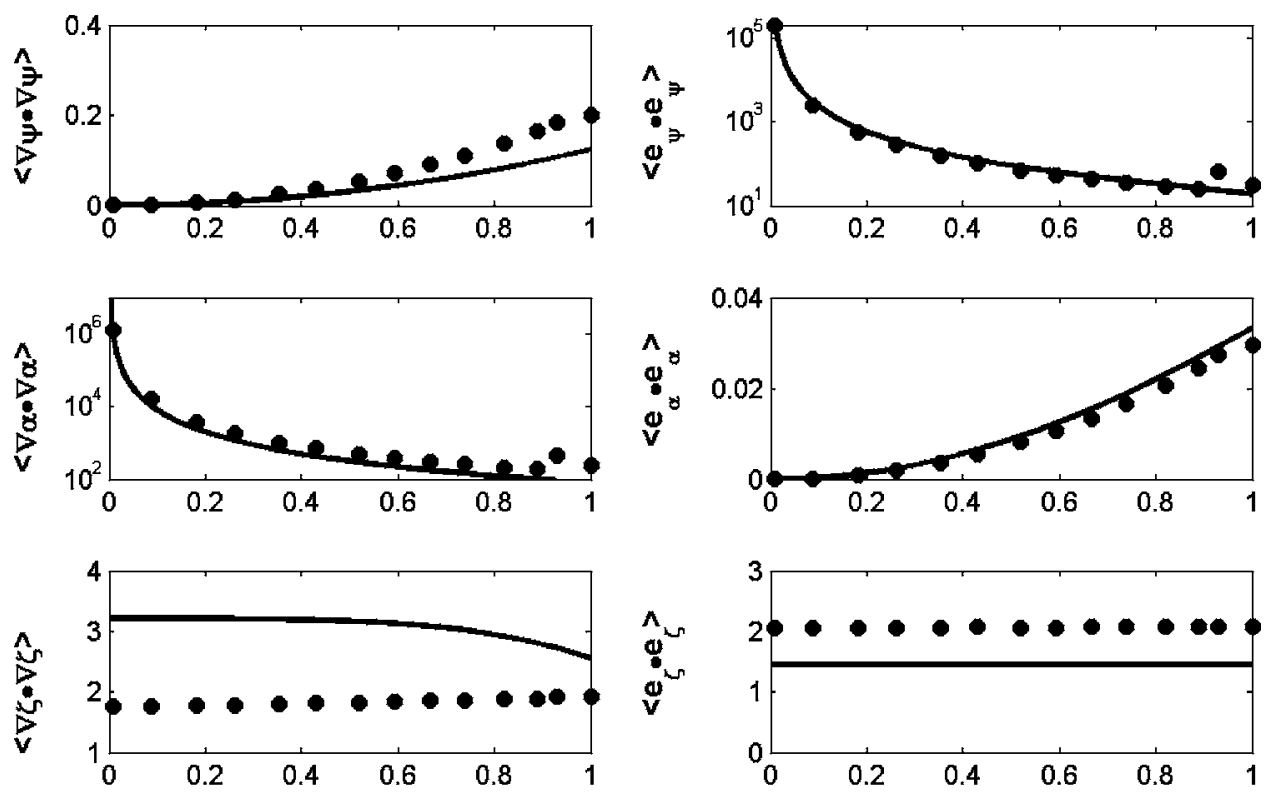

FIG. 4. Radial profile of the surface average dot product of the Hamada basis vectors computed numerically for HSX (solid circles) and compared to the analytic tokamak approximation (solid line).

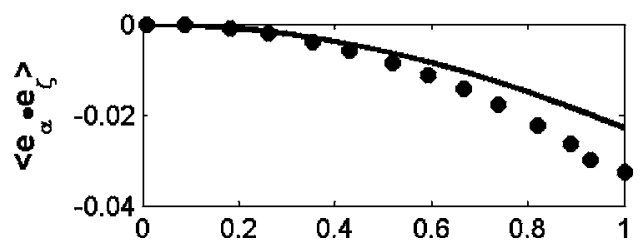



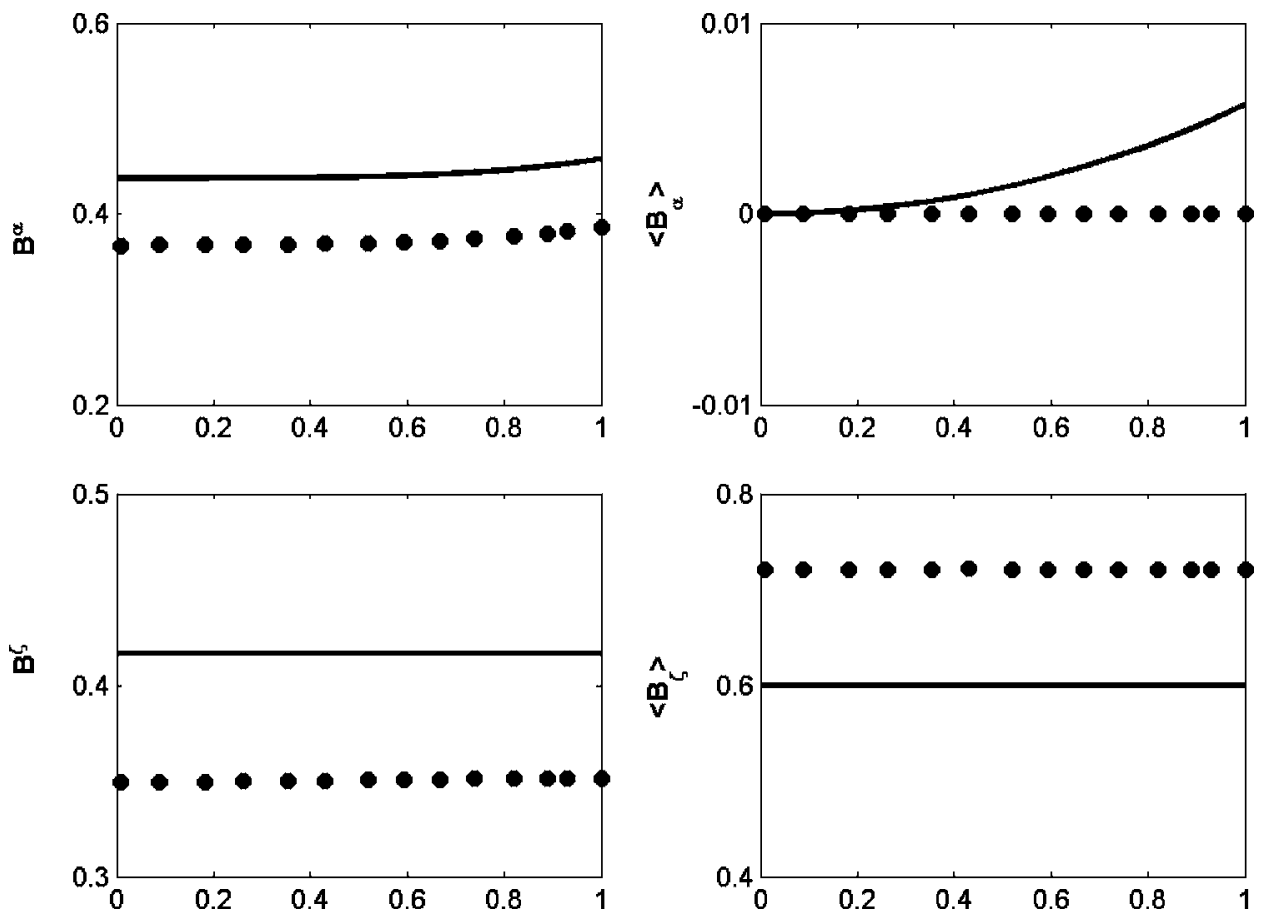

FIG. 5. Comparison of the radial profiles of the contravariant components of the magnetic field $B^{\alpha}$ and $B^{\varsigma}$ and the surface average of the covariant components $\left\langle B_{\alpha}\right\rangle$ and $\left\langle B_{\varsigma}\right\rangle$ for the numerical calculation (closed circles) with the analytic tokamak approximation (solid line). ponent $[0,1]$ in a tokamak with amplitude 0.0486 . However, in HSX the amplitude of the dominant [4,1] spectral component is 0.0734 , about $50 \%$ higher than the tokamak component.

Further insight can be gained if we write the PfirschSchlüter current as a summation of terms with respect to the Boozer toroidal and poloidal angles $\phi_{B}$ and $\theta_{B}$. As shown by
Boozer, ${ }^{19}$ if we expand the quantity $1 / B^{2}$ on a flux surface so that

$$
\frac{1}{B^{2}}=\frac{1}{B_{0}^{2}}\left(1+\sum_{n, m} \delta_{n m} \cos \left(n \phi_{B}-m \theta_{B}\right)\right),
$$
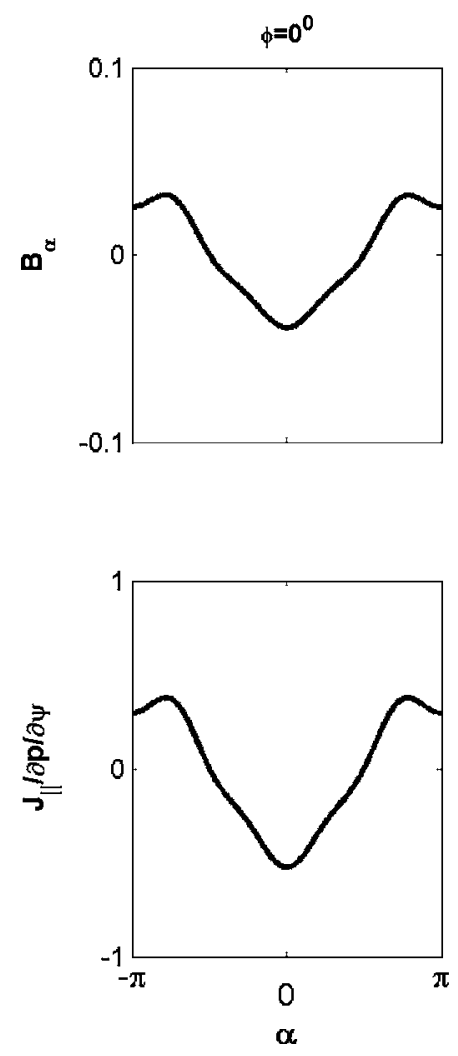
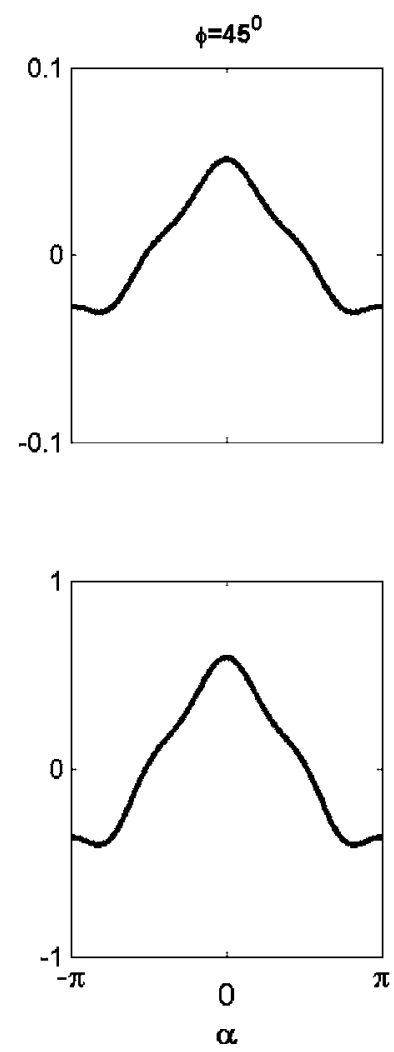

then

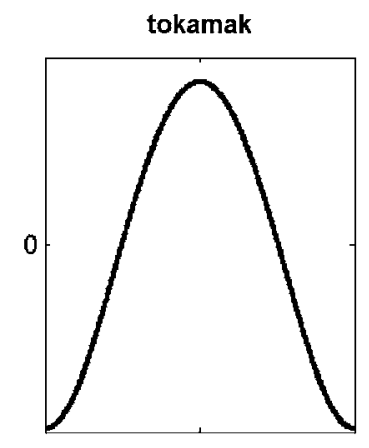

FIG. 6. Numerical calculation of $B_{\alpha}$ and $J_{\|} / d p / d \psi=\left(2 \pi B_{\alpha} / B\right)$ for HSX at $\phi=0^{\circ}$ and $\phi=45^{\circ}$ compared to the

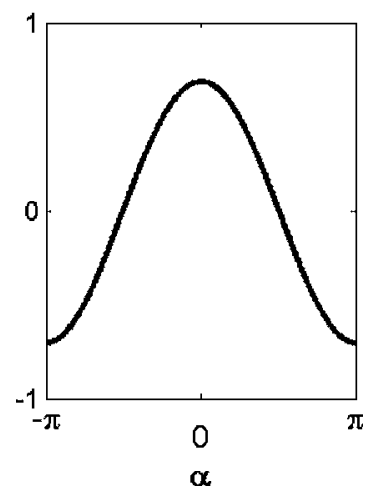



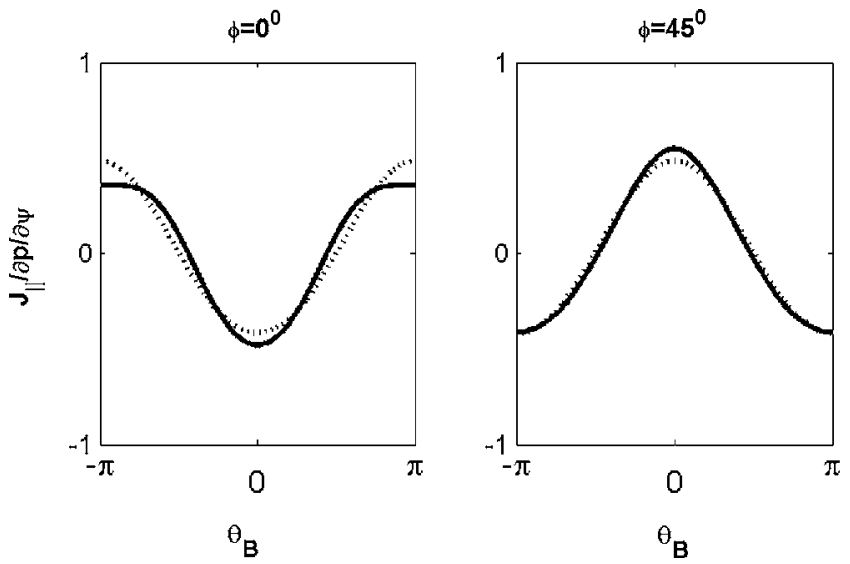

$$
J_{\|}=\frac{2 \pi B}{B_{0}^{2}} \frac{d p}{d \psi} \sum_{n, m} \frac{n I+m g}{n-m t} \delta_{n m} \cos \left(n \phi_{B}-m \theta_{B}\right),
$$

where $2 \pi I / \mu_{0}$ is the toroidal current within a flux surface and $2 \pi g / \mu_{0}$ is the poloidal current outside a flux surface. For a net current-free device like HSX, the Boozer $I$ factor is identically zero. In Eq. (32) we have kept the definition of $\psi$ to be the total toroidal flux though a magnetic surface, compared to Ref. 17 where it is defined as the flux divided by $2 \pi$. Note that there is a singularity in Eq. (32) on a rational flux surface when $t=n / m$, but we avoid this difficulty by considering only surfaces where the transform is irrational.

The advantage of writing the Pfirsch-Schlüter current in the form of Eq. (32) is that it becomes immediately apparent that the phase of the current in a quasihelically symmetric stellarator with a dominant $[4,1]$ component at the toroidal location $\phi=\pi / 4$ will be the same as in a tokamak with a $[0,1]$ magnetic field spectrum. This accounts for the similarity in the vector $\mathbf{e}_{\alpha}$ at $\phi=45^{\circ}$ with the tokamak approximation. Given the same amplitude $\delta_{n m}$, magnetic field, and rotational transform, the amplitude of the current will then scale as $g /(n-m t)$. The factor $g$ for HSX is 1.2 times larger than for a tokamak.

The normalized current is plotted in Fig. 7 for HSX at toroidal angles $0^{\circ}$ and $45^{\circ}$. For HSX, we plot the normalized current using only the dominant term in the spectrum (dotted line) as well as with the full magnetic field spectrum (solid line). In contrast to Fig. 6 where the mode amplitudes were different, we have taken the amplitude of the toroidal curvature term in Eq. (32), $\delta_{01}$, equal to the helical term for HSX $\delta_{41}$ to show more clearly the reduction of the equilibrium current in a quasihelically symmetric stellarator because of the $n-m t$ factor. Note that Figs. 6 and 7 are not exactly equivalent because in Fig. 6 , the current is plotted at constant cylindrical angle $\phi$ as a function of the Hamada angle $\alpha$, whereas in Fig. 7 the current is plotted at a constant Boozer toroidal angle $\phi_{B}$ as a function of the Boozer poloidal angle $\theta_{B}$.

One other interesting feature of these calculations is that $\left\langle B_{\alpha}\right\rangle$ and $\left\langle B_{\varsigma}\right\rangle$ are identically equal to the Boozer $I$ and $g$ factors. It is shown in Ref. 16 that $\left\langle B_{\alpha}\right\rangle$ is proportional to the toroidal current within a flux surface. For a device such as HSX with no ohmic current and sufficiently low $\beta$ that we

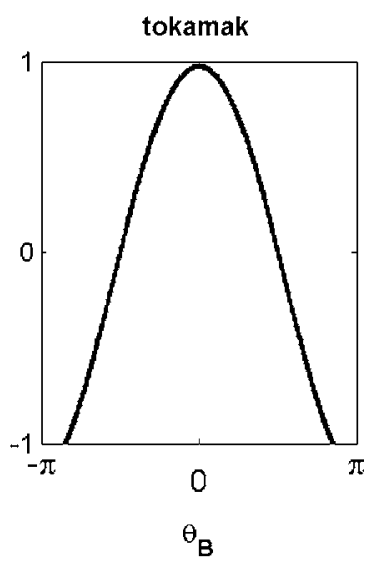

FIG. 7. Comparison of $J_{\|} / d p / d \psi$ from Eq. (32) vs the Boozer poloidal angle $\theta_{B}$ for HSX at $\phi=0^{\circ}$ and $\phi=45^{\circ}$ and for a tokamak with the same spectral amplitude. The calculation for HSX is done with just the $[4,1]$ component (dotted line), as well as with the complete spectrum (solid line), while the tokamak only has the $[0,1]$ component.

can neglect the bootstrap current, then only the vacuum magnetic field needs to be considered. In this case, $\left\langle B_{\alpha}\right\rangle$ is identically equal to zero, whereas from Eq. (29c), the tokamak analytic approximation is clearly not zero. It can be shown in a similar manner as in Ref. 16 that $\left\langle B_{\varsigma}\right\rangle$ is proportional to the poloidal current outside a flux surface. Simple use of Ampere's law for the tokamak field defined by Eq. (25) shows that the Boozer $I$ and $g$ factors defined below Eq. (32) will yield the exact same results as $\left\langle B_{\alpha}\right\rangle$ and $\left\langle B_{\mathrm{s}}\right\rangle$ in Eqs. (29c) and (29d).

In conclusion, we have developed a numerical technique to obtain the Hamada basis vectors in a three-dimensional toroidal magnetic field. We have used Nemov's method to obtain a single-valued Pfirsch-Schlüter current which ensures that the basis vectors are also single valued. The basis vectors calculated for HSX differ substantially from the large-aspect-ratio tokamak approximation simply because the magnetic field spectrum is very different between the two configurations. Differences in the surface average of the dot product of two basis vectors exist between the numerical calculations and the analytic approximation, but they are not large. One large difference, however, is in the value of $\left\langle B_{\alpha}\right\rangle$ which is identically zero in a net current-free stellarator. We have also shown that the vector $\mathbf{e}_{\alpha}$ is related to the PfirschSchlüter current and that $\left\langle B_{\alpha}\right\rangle$ and $\left\langle B_{\varsigma}\right\rangle$ are identical to the Boozer $I$ and $g$ factors. Finally, we wish to emphasize again the importance of using an accurate calculation of the Hamada basis vectors in order to compare plasma rotation measurements in an experiment to the neoclassical model.

\section{ACKNOWLEDGMENT}

This work was funded by the U.S. Department of Energy.

${ }^{1}$ S. P. Hirshman and D. J. Sigmar, Nucl. Fusion 21, 1079 (1981).

${ }^{2}$ K. C. Shaing and J. D. Callen, Phys. Fluids 26, 3315 (1983).

${ }^{3}$ M. Coronado and H. Wobig, Phys. Fluids 30, 3171 (1987).

${ }^{4}$ A. H. Boozer, Phys. Fluids 24, 1999 (1981).

${ }^{5}$ S. Hamada, Nucl. Fusion 2, 23 (1962).

${ }^{6}$ K. C. Shaing, S. P. Hirshman, and J. D. Callen, Phys. Fluids 29, 521 (1986).

${ }^{7}$ M. Coronado and J. Galindo Trejo, Phys. Fluids B 2, 530 (1990).

${ }^{8}$ M. Coronado and J. N. Talmadge, Phys. Fluids B 5, 1200 (1993).

${ }^{9}$ F. S. B. Anderson, A. F. Almagri, D. T. Anderson, P. G. Matthews, J. N. 
Talmadge, and J. L. Shohet, Fusion Technol. 27, 273 (1995).

${ }^{10}$ S. P. Gerhardt, J. N. Talmadge, J. M. Canik, and D. T. Anderson, Phys. Rev. Lett. 94, 015002 (2005); Phys. Plasmas 12, 056116 (2005).

${ }^{11}$ V. V. Nemov, Nucl. Fusion 28, 1727 (1988).

${ }^{12}$ J. N. Talmadge, V. Sakaguchi, F. S. B. Anderson, D. T. Anderson, and A. F. Almagri, Phys. Plasmas 8, 5165 (2001).

${ }^{13}$ W. D. D'haeseleer, W. N. G. Hitchon, J. D. Callen, and J. L. Shohet, Flux Coordinates and Magnetic Field Structure (Springer, Berlin, 1991).
${ }^{14}$ L. S. Solov'ev and V. D. Shafranov, in Reviews of Plasma Physics, edited by M. A. Leontovich (Consultants Bureau, New York, 1970), Vol. 5, p. 1.

${ }^{15}$ W. A. Newcomb, Phys. Fluids 2, 362 (1959).

${ }^{16}$ H. L. Berk, M. N. Rosenbluth, and J. L. Shohet, Phys. Fluids 27, 1342 (1984).

${ }^{17}$ V. V. Nemov, Nucl. Fusion 30, 927 (1990).

${ }^{18}$ M. Coronado and H. Wobig, Phys. Fluids B 4, 1294 (1992).

${ }^{19}$ A. H. Boozer, Phys. Fluids 24, 1999 (1981). 